\title{
QUASICLASSICAL ASYMPTOTICS FOR SOLUTIONS OF THE MATRIX CONJUGATION PROBLEM WITH RAPID OSCILLATION OF OFF-DIAGONAL ENTRIES
}

\author{
A. M. BUDYLIN \\ To blessed memory of my Teacher \\ Vladimir Savel'evich Buslaev
}

\begin{abstract}
The $(2 \times 2)$-matrix conjugation problem (Riemann-Hilbert problem) with rapidly oscillating off-diagonal entries is considered, along with its applications to nonlinear problems of mathematical physics. The phase function that determines oscillation is assumed to have finitely many simple stationary points and to admit power-like growth at infinity. Quasiclassical asymptotics are constructed for solutions of such a problem in the class of Hölder functions, under appropriate restrictions on the entries of the conjugation matrix. It is proved that, after separation of a certain background, the stationary points of the phase function contribute to the asymptotics additively. Along with the M. G. Kreln theory, the justification of the resulting asymptotic solutions employs the stationary phase method and the Schwarz alternating method.
\end{abstract}

\section{§1. Preliminary REMARKS}

The investigation presented in this paper was conceived by V. S. Buslaev. I am deeply grateful to him for numerous talks and advice.

Setting of the problem. We generalize some results of [1] to the case of more general matrix conjugation problems. Recall that by the conjugation problem (or the RiemannHilbert problem) we mean the following analytic factorization problem for a function on the real axis: given a function $V$ of the real argument, construct functions $H_{+}, H_{-}$ that admit analytic extenstions to the upper and lower (respectively) half-planes of the complex plane and satisfy the identity

$$
H_{+}=H_{-} V
$$

on the real axis.

In the case of the matrix conjugation problem, all function involved are assumed to be matrix-valued. We restrict ourselves to square matrices of size $2 \times 2$. We are interested in the conjugation matrices $V$ of the form

$$
V(x)=\left(\begin{array}{cc}
a(x) & b(x) e^{-i t \Phi(x)} \\
c(x) e^{i t \Phi(x)} & d(x)
\end{array}\right)
$$

where the phase function $\Phi$ is a real-valued function with finitely many simple stationary points and with power-like behavior at infinity with exponent at least 1 . The functions

2010 Mathematics Subject Classification. Primary 35Q15. Secondary 45E10, 45E99.

Key words and phrases. Matrix conjugation problem, quasiclassical asymptotics, singular integral equations, nonlinear equations of mathematical physics.

Supported by RFBR (grant no. 11-01-00458) and by Ministry of Education and Science of RF, grant nos. 8501 as of 07.09.2012, and 2012-1.5-12-000-1003-016. 
$(a-1), b, c,(d-1)$ are assumed to be complex-valued and to belong to the Schwartz class, so that the matrix $V$ turns into the identity one as $x \rightarrow \infty$. Viewing the factorization problem for the identity matrix as elementary, we see that the restrictions imposed above are of fairly general nature. In fact, we shall need to impose some additional requirements on the functions $a, b, c, d$, which will allow us to avoid certain specific issues concerning factorization of functions.

First, we assume that the condition

$$
\Delta(x) \equiv a(x) d(x)-b(x) c(x) \neq 0
$$

is fulfilled on the entire axis, and that the index of the function $\Delta$ on the closed axis is zero.

Next, denote by $k_{j}$ the stationary points of $\Phi$, put $Q=1-P$, and define $P$ to be the indicator function of the union of the intervals on which $\Phi$ grows. Assuming that the function $d$ does not vanish on the support of $P$, we complement the graph of the discontinuous function $a Q+\Delta d^{-1} P$ by line segments connecting the points $a\left(k_{j}\right)$ and $D\left(k_{j}\right) d^{-1}\left(k_{j}\right)$. It is assumed that the resulting continuous curve also has index zero (in particular, the function $a$ does not vanish on the support of $Q$ ). Recall that the index of the continuous curve described above is meant when we talk about the index of the function $a Q+\Delta d^{-1} P$.

The variable $t$ will play the role of a large positive parameter. We are interested in the asymptotic behavior of the factorizing functions $H_{ \pm}$as $t \rightarrow+\infty$, under the natural normalization condition

$$
H_{ \pm}(x) \underset{x \rightarrow \infty}{\longrightarrow} I
$$

where $I$ is the identity matrix.

We emphasize once again that, with $V$ as above and with techniques to be described below, the only substantial property is the presence of rapid oscillations in the off-diagonal entries. The case of multiple stationary points of the phase function makes no exclusion. Of course, this latter case would require exact solution of some model problems distinct from those treated in the present paper.

In the paper [1] mentioned above, the role of the phase function was played by $\Phi(x)=\frac{x^{2}}{2}$. That case serves as a base for the present considerations.

For completeness of presentation, some results of [1] will be stated below. However, we refer the reader to [1] for the history of the problem. Now we only mention the papers [7, 8], also devoted to the problem discussed here, but with substantially different line of arguments.

General characterization of the answer. The main contribution to the asymptotics as $t \rightarrow \infty$ of the factors $H_{ \pm}$is made by the stationary points of the phase function $\Phi$, i.e., the roots of the equation

$$
\Phi^{\prime}(x)=0 .
$$

After separation of some background, these contributions sum asymptotically additively. Moreover, for large $x$ the impact of the background disappears at all.

It is natural to distinguish the case of only one simple stationary point first. Importantly, this is not only the simplest case, but also a model for analysing the general case of several simple stationary points. For this simplest case, we can write the leading term of the solution's asymptotics explicitly, and also can construct asymptotic expansions complete in power orders (but not power-like), see [1].

In the case of a general phase function, we restrict ourselves to obtaining the leading asymptotic term in the space $H^{\mu, 1}(\mathbb{R})$, where $\mu>0$ is sufficiently small. Like in [1], here 
by $H^{\mu, \theta}(\mathbb{R})$ we mean the Banach space of functions with the norm

$$
\|f\|_{\mu, \theta}=\sup _{x, h \in \mathbb{R}}(1+|x|)^{\theta}\left(|f(x)|+\frac{|f(x+h)-f(x)|}{|h|^{\mu}}\right) .
$$

The precise description of the asymptotics mentioned requires some special constructions and is postponed till Theorem 3.1 .

The result obtained will be used at once for the calculation of solution asymptotics for the modified Korteveg-de Vries equation at large times.

\section{§2. Conjugation Problem with Quadratic oscillation}

In this section, for the case of the phase function $\Phi(x)=\frac{x^{2}}{2}$, we state the principal results of [1] needed in what follows. Also, once again we describe the main idea that leads to the extraction of the leading term of the asymptotics: this idea will work also in the general case.

So, at this stage we are interested in analytic factorization of a matrix $V$,

$$
H_{+}(x)=H_{-}(x) V(x), \quad V(x)=\left(\begin{array}{cc}
a & b T^{-1} \\
c T & d
\end{array}\right), \quad T=e^{i t \frac{x^{2}}{2}}, \quad T^{-1}=e^{-i t \frac{x^{2}}{2}},
$$

for large values of the parameter $t$, under the condition $H_{ \pm}(x) \rightarrow I$ as $x \rightarrow \infty$.

Heuristic arguments. The most important motivating idea can be described as follows. It is well known that if $f$ is an arbitrary smooth function decaying sufficiently fast at infinity, then the function

$$
g(x)=f(x) e^{i t x}
$$

is almost analytic in $\mathbb{C}_{+}$as $t \rightarrow+\infty$, i.e., we have the asymptotic relation

$$
p g \underset{t \rightarrow+\infty}{\sim} g
$$

where

$$
(p g)(x)=\frac{-1}{2 \pi i} \int_{\mathbb{R}} \frac{g(y) d y}{x-y+i 0},
$$

so that the operator $p$ is the orthogonal projection in $L_{2}(\mathbb{R})$ onto the subspace of functions analytic in the upper half-plane.

Similarly, the function $g$ is almost analytic in $\mathbb{C}_{-}$as $t \rightarrow-\infty$ :

$$
q g \underset{t \rightarrow-\infty}{\sim} g,
$$

where

$$
(q g)(x)=\frac{1}{2 \pi i} \int_{\mathbb{R}} \frac{g(y) d y}{x-y-i 0} .
$$

The operator $q$ is the orthogonal projection onto the subspace of functions in $L_{2}(\mathbb{R})$ that extend analytically to the lower half-plane. It is well known that $p+q=I$.

Application to quadratic exponentials. In the case where rapid oscillation comes from a quadratic exponential, the above considerations need modification. Namely, for the function $g=f T$ we still have

$$
p g \underset{t \rightarrow+\infty}{\sim} g
$$

only if the smooth function $f$ has support on the half-axis $(0,+\infty)$, and in this case we have

$$
q g \underset{t \rightarrow-\infty}{\sim} g
$$

Conversely,

$$
p g \underset{t \rightarrow-\infty}{\sim} g \text { and } q g \underset{t \rightarrow+\infty}{\sim} g
$$


whenever the support if $f$ lies on the negative half-axis.

Clearly, the reason is that the quadratic exponential $T=e^{i t \frac{x^{2}}{2}}$ extends up to a monotone decreasing function of the variable $x$ to the first and third quarters of the complex plane if $t>0$, and to the second and fourth quarters if $t<0$.

These remarks allow us to easily obtain the analytic factorization of our conjugation matrix for the first-fourth and the second-third quaters of the complex plane, respectively. To solve the factorization problem on the entire exis, we need to sew these procedures together, and such sewing requires a certain model problem, the solution of which is based on well-known aspects of analytic theory of differential equations.

Efficient factorization on half-axes. We pass from the conjugation matrix $V$ to the matrix

$$
W=\left(\begin{array}{cc}
1 & b_{1} T^{-1} \\
0 & 1
\end{array}\right)\left(\begin{array}{cc}
1 & 0 \\
c_{1} T & 1
\end{array}\right)
$$

In accordance with the above considerations, we shall treat this representation as an asymptotically efficient analytic factorization

$$
W=W_{-} W_{+}
$$

for $x>0$, as $t \rightarrow+\infty$. Note that the quadratic exponentials on the right in (2.2) admit analytic extension up to bounded functions to the first and fourth quarters of the complex plane, respectively.

The passage from $V$ to $W$ can be done via multiplication of $V$ by functions analytic in the corresponding half-planes:

$$
W=\Theta_{-} V \Theta_{+},
$$

where

$$
\Theta_{-}=\left(\begin{array}{cc}
\alpha_{-} & 0 \\
0 & \beta_{-}
\end{array}\right), \quad \Theta_{+}=\left(\begin{array}{cc}
\alpha_{+} & 0 \\
0 & \beta_{+}
\end{array}\right)
$$

and $\alpha_{ \pm}, \beta_{ \pm}$are functions that are determined uniquely (under due assumptions) by the analytic factorization relations

$$
\beta_{+} \beta_{-} d=1, \quad \alpha_{+} \alpha_{-}(a d-b c)=d
$$

and by the unit asymptotics at infinity. Indeed, it is easily seen that $W$ will take the form

$$
W=\left(\begin{array}{cc}
1+b_{1} c_{1} & b_{1} T^{-1} \\
c_{1} T & 1
\end{array}\right), \quad b_{1}=\alpha_{-} \beta_{+} b, \quad c_{1}=\alpha_{+} \beta_{-} c
$$

and can be written as in (2.2).

To present a similar formula for $x<0$, we should pass from $V$ to a matrix $W$ of the form

$$
W=\left(\begin{array}{cc}
1 & 0 \\
c_{2} T & 1
\end{array}\right)\left(\begin{array}{cc}
1 & b_{2} T^{-1} \\
0 & 1
\end{array}\right)
$$

which, for the same reasons, we may view as an aymptotically efficient analytic factorization, but now for $x<0$.

For this passage, again it suffices to multiply $V$ as in (2.3),$(2.4)$, if we redefine $\alpha_{ \pm}, \beta_{ \pm}$ by putting

$$
\alpha_{+} \alpha_{-} a=1, \quad \beta_{+} \beta_{-}(a d-b c)=a,
$$

with the unit asymptotics at infinity as before. Then

$$
c_{2}=\alpha_{+} \beta_{-} c, \quad b_{2}=\alpha_{-} \beta_{+} b .
$$


Auxiliary scalar conjugation problems. In order to join the two cases, we define the functions $\alpha_{ \pm}, \beta_{ \pm}$as solutions of the following scalar Riemann problems

$$
\begin{aligned}
\alpha_{+} \alpha_{-} & =d \Delta^{-1} P+a^{-1} Q, \quad \beta_{+} \beta_{-}=d^{-1} P+a \Delta^{-1} Q \\
\Delta & =a d-b c, \quad \alpha_{ \pm}(x), \beta_{ \pm}(x) \underset{x \rightarrow \infty}{\longrightarrow} 1
\end{aligned}
$$

where $P$ and $Q$ are the indicator functions of the half-axes $(0, \infty)$ and $(-\infty, 0)$, respectively. Recall that, by assumption, the functions $\Delta$ and $a Q+\Delta d^{-1} P$ have zero index on the axis. Then the conjugation problems (2.5) are uniquely solvable (see, e.g., [3]). Note that the identity

$$
\left(\alpha_{+} \beta_{+}\right)\left(\alpha_{-} \beta_{-}\right)=\Delta^{-1}
$$

yields an analytic factorization on the axis of the smooth function $\Delta^{-1}$. On the union of the half-axes we have

$$
W=W_{l} W_{r}, \quad W_{l}=\left(\begin{array}{cc}
1 & \gamma T^{-1} P \\
\delta T Q & 1
\end{array}\right), \quad W_{r}=\left(\begin{array}{cc}
1 & \gamma T^{-1} Q \\
\delta T P & 1
\end{array}\right)
$$

where $\gamma=\alpha_{-} \beta_{+} b, \delta=\alpha_{+} \beta_{-} c$.

Clearly, at the point zero this representation of the matrix $W$ cannot serve as an appoximation to analytic factorization, because the conjuntion functions for the auxiliary scalar problems have a jump at zero. To correct this expansion, from each factor $W_{l}$, $W_{r}$ in (2.6) we extract its asymptotics at zero (for $x=0$ ), and then find a precise analytic factorization $Y_{-} Y_{+}$for the product $W_{0}$ of these two asymptotics. The remaining additional embordering factors, call them $L$ and $R$, turn out to be efficiently analytic in the corresponding half-planes. Schematically, this procedure is presented by the passages

$$
V \mapsto W=W_{l} W_{r}=L W_{0} R=L\left(Y_{-} Y_{+}\right) R=\left(L Y_{-}\right)\left(Y_{+} R\right)
$$

The asymptotics of $W_{l}, W_{r}$. Putting

$$
\begin{aligned}
& \nu_{0}=\frac{-1}{2 \pi} \ln \left(a_{0} d_{0} \Delta_{0}^{-1}\right), \quad a_{0}=a(0), \quad b_{0}=b(0), \\
& c_{0}=c(0), \quad d_{0}=d(0), \quad \Delta_{0}=\Delta(0),
\end{aligned}
$$

we arrive at the following asymptotic expansions:

$$
\begin{aligned}
\ln \alpha_{+}(x) & =\frac{-1}{2 \pi i} \int_{-\infty}^{0} \frac{-\ln a(y) d y}{x-y+i 0}+\frac{-1}{2 \pi i} \int_{0}^{\infty} \frac{\ln \left[d(y) \Delta^{-1}(y)\right] d y}{x-y+i 0} \\
& \underset{x \rightarrow 0}{\sim}-i \nu_{0} \ln (x+i 0)+A_{+}+\sum_{\substack{k \geq 1 \\
0 \leq j \leq k}} A_{k j}^{+} x^{k} \ln ^{j}(x+i 0) .
\end{aligned}
$$

Here $\ln (x+i 0)$ is the principal branch of the logarithm with cut along the positive semiaxis. Similarly,

$$
\begin{aligned}
\ln \alpha_{-}(x) & =\frac{1}{2 \pi i} \int_{-\infty}^{0} \frac{-\ln a(y) d y}{x-y-i 0}+\frac{1}{2 \pi i} \int_{0}^{\infty} \frac{\ln \left[d(y) \Delta^{-1}(y)\right] d y}{x-y-i 0} \\
& \underset{x \rightarrow 0}{\sim} i \nu_{0} \ln (x-i 0)+A_{-}+\sum_{\substack{k \geq 1 \\
0 \leq j \leq k}} A_{k j}^{-} x^{k} \ln ^{j}(x-i 0)
\end{aligned}
$$


and

$$
\begin{aligned}
& \ln \beta_{+}(x) \underset{x \rightarrow 0}{\sim} i \nu_{0} \ln (x+i 0)+B_{+}+\sum_{\substack{k \geq 1 \\
0 \leq j \leq k}} B_{k j}^{+} x^{k} \ln ^{j}(x+i 0), \\
& \ln \beta_{-}(x) \underset{x \rightarrow 0}{\sim}-i \nu_{0} \ln (x+i 0)+B_{-}+\sum_{\substack{k \geq 1 \\
0 \leq j \leq k}} B_{k j}^{-} x^{k} \ln ^{j}(x-i 0) .
\end{aligned}
$$

Thus,

$$
\alpha_{ \pm}(x) \underset{x \rightarrow 0}{\sim} \sigma_{ \pm}(x)=(x \pm i 0)^{\mp i \nu_{0}} e^{A_{ \pm}}, \quad \beta_{ \pm}(x) \underset{x \rightarrow 0}{\sim} \tau_{ \pm}(x)=(x \pm i 0)^{ \pm i \nu_{0}} e^{B_{ \pm}},
$$

where $(x \pm i 0)^{ \pm i \nu_{0}}=e^{ \pm i \nu_{0} \ln (x \pm i 0)}$, and the functions $\ln (x \pm i 0)$ are the principal branches of the logarithm with cut along the positive semiaxis. Observe that

$$
\sigma_{+} \sigma_{-}=d_{0} \Delta_{0}^{-1} P+a_{0}^{-1} Q, \quad \tau_{+} \tau_{-}=d_{0}^{-1} P+a_{0} \Delta_{0}^{-1} Q .
$$

As a consequence, $\gamma(x) \underset{x \rightarrow 0}{\sim} \gamma_{0}(x)$, where

$$
\begin{aligned}
& \gamma_{0}=b_{0} \sigma_{-} \tau_{+}=b_{0}(x-i 0)^{i \nu_{0}}(x+i 0)^{i \nu_{0}} e^{A_{-}+B_{+}}, \\
& \delta_{0}=c_{0} \sigma_{+} \tau_{-}=b_{0}(x-i 0)^{-i \nu_{0}}(x+i 0)^{-i \nu_{0}} e^{A_{+}+B_{-}} .
\end{aligned}
$$

Thus, the asymptotics of $W$ at zero has the form

$$
\begin{aligned}
W_{0} & =\left(\begin{array}{cc}
1 & \gamma_{0} T^{-1} P \\
\delta_{0} T Q & 1
\end{array}\right)\left(\begin{array}{cc}
1 & \gamma_{0} T^{-1} Q \\
\delta_{0} T P & 1
\end{array}\right) \\
& =\left(\begin{array}{cc}
1+\gamma_{0} \delta_{0} P & \gamma_{0} T^{-1} \\
\delta_{0} T & 1+\gamma_{0} \delta_{0} Q
\end{array}\right)=\Omega_{-} V_{0} \Omega_{+},
\end{aligned}
$$

where

$$
\Omega_{ \pm}=\left(\begin{array}{cc}
\sigma_{ \pm} & 0 \\
0 & \tau_{ \pm}
\end{array}\right), \quad V_{0}=\left(\begin{array}{cc}
a_{0} & b_{0} T^{-1} \\
c_{0} T & d_{0}
\end{array}\right)
$$

This representation leads us to the model matrix conjugation problem with the conjugation function $V_{0}$. This model problem involves no slow dependence, and should admit exact solution.

Solution of the model problem. Our model problem has the form

$$
G_{+}=G_{-} V_{0} .
$$

Its solutions are well known due to its relationship with the ordinary differential equation for the harmonic oscillator. We must only select the solutions with proper asymptotic behavior. Referring to [1] for the details, here we write the solution for $t=1$ in terms of the standard functions of the parabolic cylinder (Weber functions):

$$
\left(G_{-}\right)_{12}=e^{-i \frac{x^{2}}{4}}\left(C_{1} D_{\nu}\left(e^{i \frac{\pi}{4}} x\right)+C_{2} D_{\nu}\left(-e^{i \frac{\pi}{4}} x\right)\right) .
$$

Here

$$
\nu=i \nu_{0}, \quad C_{2}=-C_{1} e^{-i \pi \nu}, \quad C_{1}=-\frac{a_{0} d_{0}}{c_{0} \Delta_{0}} t^{-i \frac{\nu_{0}}{2}} e^{-A_{+}+\frac{\pi}{4} \nu_{0}} .
$$

The function $G_{22}$ is determined by the relations

$$
G_{12}^{\prime}+i x G_{12}=\lambda G_{22}, \quad \lambda=\frac{a_{0} d_{0} \sqrt{2 \pi}}{c_{0} \Delta_{0} \Gamma\left(-i \nu_{0}\right)} e^{i \frac{\pi}{4}-\frac{\pi}{2} \nu_{0}-A_{+}-B_{-}} .
$$

Similarly,

$$
\left(G_{-}\right)_{21}=e^{i \frac{x^{2}}{4}} E_{1} D_{\nu-1}\left(e^{i \frac{\pi}{4}} x\right), \quad E_{1}=-\frac{c_{0} \Gamma\left(1-i \nu_{0}\right)}{\Delta_{0} \sqrt{2 \pi}} t^{i \frac{\nu_{0}}{2}} e^{\frac{3 \pi}{4} \nu_{0}-B_{+}},
$$


and $G_{11}$ is defined by

$$
G_{21}^{\prime}-i x G_{21}=\mu G_{11}, \quad \lambda \mu=\nu_{0} .
$$

Embordering factors. Concerning the differences

$$
\gamma_{\infty}=\gamma-\gamma_{0}, \quad \delta_{\infty}=\delta-\delta_{0}
$$

the following estimates were proved in [1]:

$$
p\left(\gamma_{\infty} T^{-1} P\right)=O\left(\frac{t^{-i \nu_{0}} \ln t}{\sqrt{t}(1+|x| \sqrt{t})}\right), \quad p\left(\delta_{\infty} T Q\right)=O\left(\frac{t^{-i \nu_{0}} \ln t}{\sqrt{t}(1+|x| \sqrt{t})}\right) .
$$

Similarly,

$$
q\left(\gamma_{\infty} T^{-1} Q\right)=O\left(\frac{t^{i \nu_{0}} \ln t}{\sqrt{t}(1+|x| \sqrt{t})}\right), \quad q\left(\delta_{\infty} T P\right)=O\left(\frac{t^{i \nu_{0}} \ln t}{\sqrt{t}(1+|x| \sqrt{t})}\right) .
$$

Put

$$
\begin{array}{ll}
L=\left(\begin{array}{cc}
1 & \gamma_{\infty} T^{-1} P \\
\delta_{\infty} T Q & 1
\end{array}\right), \quad R=\left(\begin{array}{cc}
1 & \gamma_{\infty} T^{-1} Q \\
\delta_{\infty} T P & 1
\end{array}\right), \\
L_{-}=q(L), & R_{+}=p(R) .
\end{array}
$$

Estimates (2.11) and (2.12) show that the matrix $L$ is almost analytic in $\mathbb{C}_{-}$, and $R$ is almost analytic in $\mathbb{C}_{+}$; therefore, we have

$$
L \approx L_{-}, \quad R \approx R_{+}
$$

up to quantities of order

$$
O\left(\frac{t^{ \pm i \nu_{0}} \ln t}{\sqrt{t}(1+|x| \sqrt{t})}\right)
$$

Properties of triangular matrices allow us to white $W_{l}$ and $W_{r}$ in the form

$$
W_{l}=L\left(\begin{array}{cc}
1 & \gamma_{0} T^{-1} P \\
\delta_{0} T Q & 1
\end{array}\right), \quad W_{r}=\left(\begin{array}{cc}
1 & \gamma_{0} T^{-1} Q \\
\delta_{0} T P & 1
\end{array}\right) R
$$

in full agreement with (2.7), 2.10).

If we replace the almost analytic factors $L$ and $R$ with the corresponding analytic functions $L_{-}$and $R_{+}$, then, by (2.11) and (2.12), the error will be of order

$$
O\left(\frac{t^{\left|\operatorname{Re}\left(i \nu_{0}\right)\right|-1 / 2} \ln t}{1+\sqrt{t}|x|}\right) .
$$

This error can be leveled by the discrepancy method, see [1].

Asymptotics of $H$. Now, the asymptotics as $t \rightarrow+\infty$ of the matrix $H$ is given by

$$
H_{-} \sim G_{-}\left(\begin{array}{cc}
\sigma_{-}^{-1} & 0 \\
0 & \tau_{-}^{-1}
\end{array}\right) L_{-}^{-1}\left(\begin{array}{cc}
\alpha_{-} & 0 \\
0 & \beta_{-}
\end{array}\right) \text {. }
$$

For example, for $|x|$ separated away from zero, we find

$$
H_{-} \sim\left(\begin{array}{cc}
\alpha_{-}+\alpha_{-} \tau_{-}^{-1} G_{12} \delta_{0} T Q & \beta_{-} \tau_{-}^{-1} G_{12}+\beta_{-} \gamma_{0} T^{-1} P \\
\alpha_{-} \sigma_{-}^{-1} G_{21}+\alpha_{-} \delta_{0} T Q & \beta_{-}+\beta_{-} \sigma_{-}^{-1} G_{21} \gamma_{0} T^{-1} P
\end{array}\right) .
$$

We write the leading term of this asymptotics for large $|x|$ for the entry $\left(H_{-}\right)_{12}$. Precisely this asymptotics is most important in applications to nonlinear equations of mathematical physics. We have

$$
\left(H_{-}\right)_{12} \sim \frac{a_{0} d_{0}}{c_{0} \Delta_{0}} \frac{\sqrt{2 \pi}}{\Gamma\left(-i \nu_{0}\right)} t^{-\frac{1}{2}-i \nu_{0}} e^{-A_{+}-B_{-}} e^{-i \frac{\pi}{4}} e^{-\frac{\pi \nu_{0}}{2}} \cdot \frac{1}{x} .
$$


So, the following statement is one of the results of [1].

Theorem 2.1. Under the conditions imposed above on the entries of the conjugation matrix, for any sufficiently large $t>0$ the solution of the conjugation problem (2.1) exists and has asymptotics determined by relations (2.15), (2.16), and (2.17) with discrepancy $O\left(t^{\left|\operatorname{Im} \nu_{0}\right|-1+\varepsilon}\right)$ for an arbitrarily small $\varepsilon>0$ relative to $H^{\mu, 1}(\mathbb{R})$, where $\mu>0$ is sufficiently small.

For what follows we note that, to justify the results in [1, a relationship was employed between the transposed conjugation problem

$$
H_{+}^{T}=V^{T} H_{-}^{T}, \quad H_{ \pm}^{T} \underset{x \rightarrow \infty}{\sim} I,
$$

and the inversion problem for the singular operator $I-F^{T} q$, where $F$ is the operator of multiplication by the matrix-valued function $F(x)=I-V$, and $F^{T}$ is the operator of multiplication by the transpose matrix.

Namely, if we define a matrix-valued function $K^{T}$ as a solution of the equation

$$
\left(I-F^{T} q\right) K^{T}=F^{T},
$$

then the transposed conjugation problem has a solution of the form

$$
H_{+}^{T}=I-p\left(K^{T}\right), \quad H_{-}^{T}=I+q\left(K^{T}\right) .
$$

As a consequence, we find

$$
H_{+}=I-p(K), \quad H_{-}=I+q(K) .
$$

If a pair of factors $S_{ \pm}$determines a solution $K_{0}^{T}$ of the equation

$$
\left(I-F_{0}^{T} q\right) K_{0}^{T}=F_{0}^{T}, \quad F_{0}=S_{+}^{-1} S_{-},
$$

then for the perturbed conjugation matrix $V=I+F_{0}+\mathcal{E}$ we get an equation for $K^{T}$ :

$$
\left(I+F_{0}^{T} q+\mathcal{E}^{T} q\right) K^{T}=F_{0}^{T}+\mathcal{E}^{T},
$$

which can be solved by iteration provided the perturbation $\mathcal{E}$ is appropriately small.

\section{§3. Asymptotics of solutions of the Conjugation problem IN THE GENERAL CASE}

\subsection{Preparing separation of contributions.}

Idea of generalization. We turn to the general case of analytic factorization of the matrix-valued function

$$
V(x)=\left(\begin{array}{cc}
a(x) & b(x) e^{-i t \Phi(x)} \\
c(x) e^{i t \Phi(x)} & d(x)
\end{array}\right) .
$$

The preceding considerations generalize to the case of an arbitrary smooth phase function $\Phi$ that has finitely many simple stationary points and a power-like growth at infinity with an exponent at least 1 . Here, the central question will be about separation of the contributions of individual stationary points.

We split the procedure of separating the contributions into several steps.

First, we solve an auxiliary scalar problem, arriving at an efficient (in the same sense as in (2.60) analytic factorization outside of the stationary points of the phase function.

At the next step, we find the asymptotic behavior of the efficient factorizing functions (denoting them $U_{l}$ and $U_{r}$ this time) at the stationary points. However, unlike the case of the quadratic phase function, now we preserve the local nature of these asymptotic formulas by involving standard cut-off functions in order to exclude interference of contributions of stationary points. Like in the case of the quadratic phase function, the 
supplementary factors will be described as the embordering functions $L$ and $R$ (cf. (2.7)). These factors will be efficiently analytic in the corresponding half-planes.

The third step is specific for separation of contributions. It consists in analytic factorization of the diagonal of the conjugation matrix obtained at the preceding step. It is aimed at reducing the problem to a form adjusted to application of the alternating Schwarz method, which is the standard algebraic procedure for summation of contributions.

At the fourth step, we use the Schwarz method to fustify the summation of contributions made by the stationay points of the phase function. However, to obtain explicit formulas we shall need yet another step (a step back, a sort of "defactorization" of the diagonal of the conjugation matrix), because the procedure of summation of contributions and that of analytic factorization of the model problem with the quadratic phase function are done for matrices of different structures, which requires an elementary recalculation.

Schematically, all this process is presented by the passages

$$
\begin{aligned}
V \mapsto \Theta_{-} V \Theta_{+} & =U_{l} U_{r}=L \prod V_{i} R=L \Psi_{-} W \Psi_{+} R \\
& \cong L \Psi_{-}\left(I-\sum N_{i-}\right)\left(I+\sum N_{i+}\right) \Psi_{+} R \cong U_{-} U_{+}
\end{aligned}
$$

and is described below in more detail.

Step 1: the auxiliary scalar conjugation problem. We denote by $k_{i}$ the stationary points of the phase function $\Phi$ and by $P$ the indicator function for the union of the intervals on which $\Phi$ is monotone increasing. Put $Q=1-P$.

Following the line described above, we introduce auxiliary scalar analytic factorizations

$$
\begin{aligned}
\alpha_{+} \alpha_{-} & =d \Delta^{-1} P+a^{-1} Q, \quad \beta_{+} \beta_{-}=d^{-1} P+a \Delta^{-1} Q \\
\Delta & =a d-b c, \quad \alpha_{ \pm}(x), \beta_{ \pm}(x) \underset{x \rightarrow \infty}{\longrightarrow} 1 .
\end{aligned}
$$

Again, we observe the identity

$$
\left(\alpha_{+} \beta_{+}\right)\left(\alpha_{-} \beta_{-}\right)=\Delta^{-1}
$$

As before,

$$
\begin{aligned}
W & =\Theta_{-} V \Theta_{+}=\left(\begin{array}{cc}
1 & \gamma T^{-1} P \\
\delta T Q & 1
\end{array}\right)\left(\begin{array}{cc}
1 & \gamma T^{-1} Q \\
\delta T P & 1
\end{array}\right), \\
\gamma & =\alpha_{-} \beta_{+} b, \quad \delta=\alpha_{+} \beta_{-} c
\end{aligned}
$$

where $\Theta_{ \pm}$are the matrices defined in (2.4). We put

$$
\begin{aligned}
& \nu_{j}=\frac{-1}{2 \pi} \ln \left(a_{j} d_{j} \Delta_{j}^{-1}\right), \quad a_{j}=a\left(k_{j}\right), \quad b_{j}=b\left(k_{j}\right), \\
& c_{j}=c\left(k_{j}\right), \quad d_{j}=d\left(k_{j}\right), \quad \Delta_{j}=\Delta\left(k_{j}\right) .
\end{aligned}
$$

We assume additionally that the parameters $\nu_{j}$ are real. This assumption is not principal, but will allow us to treat the contributions of different stationary points as quantities of one order of smallness. Precisely this case is most important in applications.

The set $Q(\mathbb{R})$ on which the phase function is monotone decreasing is a union of disjoint intervals (including, possibly, infinite intervals). The points $k_{j}$ are ends of these intervals. If a point $k_{j}$ is the right end of an interval in the system $Q(\mathbb{R})$, i.e., $k_{i}$ is a point of 
minimum, then, as before, we put

$$
\begin{aligned}
& \ln \alpha_{+}(x)=\frac{-1}{2 \pi i} \int_{\mathbb{R}} \frac{-\ln a(y) Q(y) d y}{x-y+i 0}+\frac{-1}{2 \pi i} \int_{\mathbb{R}} \frac{\ln \left[d(y) \Delta^{-1}(y) P(y)\right] d y}{x-y+i 0} \\
& \underset{x \rightarrow k_{j}}{\sim}-i \nu_{j} \ln \left(x-k_{j}+i 0\right)+A_{j+} .
\end{aligned}
$$

Here, again, $\ln (x+i 0)$ is the principal branch of the logarithm with cut along the positive semiaxis. Similarly,

$$
\begin{aligned}
& \ln \alpha_{-}(x)=\frac{1}{2 \pi i} \int_{\mathbb{R}} \frac{-\ln a(y) Q(y) d y}{x-y-i 0}+\frac{1}{2 \pi i} \int_{\mathbb{R}} \frac{\ln \left[d(y) \Delta^{-1}(y) P(y)\right] d y}{x-y-i 0} \\
& \underset{x \rightarrow k_{j}}{\sim} i \nu_{j} \ln \left(x-k_{j}-i 0\right)+A_{j-} .
\end{aligned}
$$

Also, we have

$$
\ln \beta_{+}(x) \underset{x \rightarrow k_{j}}{\sim} i \nu_{j} \ln \left(x-k_{j}+i 0\right)+B_{j+}, \quad \ln \beta_{-}(x) \underset{x \rightarrow k_{j}}{\sim}-i \nu_{j} \ln \left(x-k_{j}-i 0\right)+B_{j-} .
$$

If $k_{j}$ is the left end of an interval in the system $Q(\mathbb{R})$, i.e., $k_{j}$ is a point of maximum, then, for the same choice of a branch the logarithm, we have

$$
\ln \alpha_{+}(x) \underset{x \rightarrow k_{j}}{\sim} i \nu_{j} \ln \left(x-k_{j}+i 0\right)+A_{j+}, \quad \ln \alpha_{-}(x) \underset{x \rightarrow k_{j}}{\sim}-i \nu_{j} \ln \left(x-k_{j}-i 0\right)+A_{j-},
$$

and, similarly,

$$
\ln \beta_{+}(x) \underset{x \rightarrow k_{j}}{\sim}-i \nu_{j} \ln \left(x-k_{j}+i 0\right)+B_{j+}, \quad \ln \beta_{-}(x) \underset{x \rightarrow k_{j}}{\sim} i \nu_{j} \ln \left(x-k_{j}-i 0\right)+B_{j-} .
$$

We shall not present precise expressions for $A_{j \pm}$ and $B_{j \pm}$, viewing the above asymptotic relations as the definitions of these constants.

In what follows, we call the points $k_{j}$ the left and right turning points.

Step 2: finding the asymptotic behavior at the stationary points. Our goal is to modify the expansion (3.3) in a neighborhood of the stationary points of the phase function $\Phi$ so as to ensure the applicability of the discrepancy method for obtaining the exact analytic factorization.

So, in the case of a minumum point $k_{j}$ we have

$$
\begin{gathered}
\alpha_{j \pm}(x) \underset{x \rightarrow k_{j}}{\sim} \sigma_{j \pm}(x)=\left(x-k_{j}+i 0\right)^{\mp i \nu_{j}} e^{A_{j \pm}}, \\
\beta_{j \pm}(x) \underset{x \rightarrow k_{j}}{\sim} \tau_{j \pm}(x)=\left(x-k_{j}-i 0\right)^{ \pm i \nu_{j}} e^{B_{j \pm}},
\end{gathered}
$$

where, as before, $(x \pm i 0)^{\mp i \nu_{j}}=e^{\mp i \nu_{j} \ln (x \pm i 0)}$, and the functions $\ln (x \pm i 0)$ are the principal branches of the logarithm with cut along the positive semiaxis. In the case of a point of maximum,

$$
\begin{aligned}
& \alpha_{j \pm}(x) \underset{x \rightarrow k_{j}}{\sim} \sigma_{j \pm}(x)=\left(x-k_{j}+i 0\right)^{ \pm i \nu_{j}} e^{A_{j \pm}} \\
& \beta_{j \pm}(x) \underset{x \rightarrow k_{j}}{\sim} \tau_{j \pm}(x)=\left(x-k_{j}-i 0\right)^{\mp i \nu_{j}} e^{B_{j \pm}} .
\end{aligned}
$$

In any case,

$$
\sigma_{j+} \sigma_{j-}=d_{j} \Delta_{j}^{-1} P_{j}+a_{j}^{-1} Q_{j}, \quad \tau_{j+} \tau_{j-}=d_{j}^{-1} P_{j}+a_{j} \Delta_{j}^{-1} Q_{j},
$$

where $P_{j}+Q_{j}=1$, and $P_{j}$ is the indicator function of the real semiaxis ending at $k_{j}$ and coinciding with $P$ near $k_{j}$.

It follows that $\gamma(x) \underset{x \rightarrow k_{j}}{\sim} \gamma_{j}(x)$ and $\delta(x) \underset{x \rightarrow k_{j}}{\sim} \delta_{j}(x)$, where

$$
\gamma_{j}=b_{j} \sigma_{j-} \tau_{j+}, \quad \delta_{j}=c_{j} \sigma_{j+} \tau_{j-} .
$$


Now, we additionally split every factor on the right in (3.3), extracting the asymptotic behavior in neighborhoods of the stationary points:

$$
\left(\begin{array}{cc}
1 & \gamma T^{-1} P \\
\delta T Q & 1
\end{array}\right) \underset{k \rightarrow k_{j}}{\sim} V_{j}^{-}=\left(\begin{array}{cc}
1 & \gamma_{j} \chi_{j} T_{j}^{-1} P \\
\delta_{j} \chi_{j} T_{j} Q & 1
\end{array}\right)
$$

and

$$
\left(\begin{array}{cc}
1 & \gamma T^{-1} Q \\
\delta T P & 1
\end{array}\right) \underset{k \rightarrow k_{j}}{\sim} V_{j}^{+}=\left(\begin{array}{cc}
1 & \gamma_{j} \chi_{j} T_{j}^{-1} Q \\
\delta_{j} \chi_{j} T_{j} P & 1
\end{array}\right) .
$$

Here, in a neighborhood of a turning point $k_{j}$ we put

$$
T \sim T_{j},
$$

where

$$
T_{j}(x)=e^{i t \Phi\left(k_{j}\right)} e^{i t \frac{\Phi^{\prime \prime}\left(k_{j}\right)}{2}\left(x-k_{j}\right)^{2}},
$$

and $\chi_{j}$ denotes a smooth cut-off function equal to 1 near $k_{j}$. The supports of $\chi_{j}$ are assumed to be disjoint, which implies that the matrices $V_{j}$ commute, as well as the matrices $V_{j}^{+}$. Also, the matrices $V_{j}^{-}$and $V_{k}^{+}$commute for different indices $j$ and $k$.

We obtain the expansions

$$
U_{l}=\left(\begin{array}{cc}
1 & \gamma T^{-1} P \\
\delta T Q & 1
\end{array}\right)=L \prod_{j}\left(\begin{array}{cc}
1 & \gamma_{j} \chi_{j} T_{j}^{-1} P \\
\delta_{j} \chi_{j} T_{j} Q & 1
\end{array}\right)
$$

and

$$
U_{r}=\left(\begin{array}{cc}
1 & \gamma T^{-1} Q \\
\delta T P & 1
\end{array}\right)=\prod_{j}\left(\begin{array}{cc}
1 & \gamma_{j} \chi_{j} T_{j}^{-1} Q \\
\delta_{j} \chi_{j} T_{j} P & 1
\end{array}\right) R .
$$

Like in the case of one turning point, the factors $L$ and $R$ are almost analytic, respectively, in $\mathbb{C}_{-}$and $\mathbb{C}_{+}$, are close to 1 with an error of $O\left(t^{-1 / 2} \ln t(1+\sqrt{t}|x|)^{-1}\right)(\operatorname{cf} .(2.14)$ ), and can be reshaped into strictly analytic functions with the help of the discrepancy method (see Subsection 2.5 in [1]). These facts can be proved precisely as it was done in the proof of Theorem 2.2 in [1].

Step 3: factorization of the diagonal. At the preceding step we arrived at the following factorization:

$$
\Theta_{-} V \Theta_{+}=L \prod V_{j} R
$$

where

$$
V_{j}=V_{j}^{-} V_{j}^{+}=\left(\begin{array}{cc}
1+\gamma_{i} \delta_{i} \chi_{j}^{2} P & \gamma_{j} \chi_{j} T_{j}^{-1} \\
\delta_{j} \chi_{j} T_{j} & 1+\gamma_{j} \delta_{j} \chi_{j}^{2} Q
\end{array}\right)
$$

and

$$
\prod V_{j}=\left(\begin{array}{cc}
1+\sum \gamma_{j} \delta_{j} \chi_{j}^{2} P & \sum \gamma_{j} \chi_{j} T_{j}^{-1} \\
\sum \delta_{j} \chi_{j} T_{j} & 1+\sum \gamma_{j} \delta_{j} \chi_{j}^{2} Q
\end{array}\right)
$$

It should be noted that the asymptotics of the factorizing functions of the matrix $\prod V_{j}$ depends, in the senior order, only on the asymptotic behavior of the functions $\gamma_{j}, \delta_{j}$ at the stationary points of the phase function $\Phi$, which can be seen from the presence of the cut-off functions $\chi_{j}$ in the definiton of $\prod V_{j}$.

Factorization of the diagonal of the matrix $\prod V_{j}$ will lead us to a conjugation problem in which the contributions of the stationary points sum asymptotically additively.

To show this, we need some auxiliary calculations. Namely, we introduce the scalar analytic factorizations $\varphi_{j-} \varphi_{j+}=1+\gamma_{j} \delta_{j} \chi_{j}^{2} P$ and $\psi_{j-} \psi_{j+}=1+\gamma_{j} \delta_{j} \chi_{j}^{2} Q$. The functions 
$\varphi_{j \pm}(x), \psi_{j \pm}(x)$ are normalized by the requirement that they tend to 1 as $x \rightarrow \infty$. We put

$$
\varphi_{ \pm}=\prod \varphi_{j \pm}, \quad \psi_{ \pm}=\prod \psi_{j \pm}
$$

and

$$
\Psi_{-}=\left(\begin{array}{cc}
\varphi_{-} & 0 \\
0 & \psi_{-}
\end{array}\right), \quad \Psi_{+}=\left(\begin{array}{cc}
\varphi_{+} & 0 \\
0 & \psi_{+}
\end{array}\right)
$$

We have the identity

$$
\Psi_{-} \Psi_{+}=\left(\begin{array}{cc}
1+\sum \gamma_{j} \delta_{j} \chi_{j}^{2} P & 0 \\
0 & 1+\sum \gamma_{j} \delta_{j} \chi_{j}^{2} Q
\end{array}\right),
$$

which is an analytic factorization of the diagonal of the matrix $\prod V_{j}$. It follows that

$$
\prod V_{j}=\Psi_{-} W \Psi_{+}
$$

where we have put

$$
W=I-F, \quad F=\sum F_{j}, \quad F_{j}=-\left(\begin{array}{cc}
0 & f_{j} T_{j}^{-1} \\
h_{j} T_{j} & 0
\end{array}\right) .
$$

Here

$$
f_{j}=\gamma_{j} \chi_{j} \varphi_{-}^{-1} \psi_{+}^{-1}, \quad h_{j}=\delta_{j} \chi_{j} \varphi_{+}^{-1} \psi_{-}^{-1} .
$$

We shall show that the conjugation problem for the matrix $W$ reduces asymptotically to the additive summation of the contributions of the conjugation problems for the matrices $W_{j}=I-F_{j}$ in a domain not containing neighborhoods of the turning points.

\subsection{Separation of contributions.}

Alternating Schwarz method. Before passing to the fourth step, we recall the general algebraic scheme of the alternating Schwarz method. The details of this interpretation can be found in 2]. This method concerns an algebraic construction of the operator inverse to $I-\sum G_{i}$ provided that we know the inverses to the operators $I-G_{i}$.

Let $G_{i}(i=1, \ldots n)$ be linear operators in a complex vector space $\mathcal{X}$. Assume that all operators $I-G_{i}$ are bijective. Put

$$
I-\Gamma_{i}=\left(I-G_{i}\right)^{-1}
$$

and

$$
G=\sum_{i=1}^{n} G_{i} .
$$

The essence of the alternating Schwarz method will be shown in a series of claims.

First. If an operator matrix

$$
\mathbf{\Phi}=\left(\begin{array}{cccc}
I & \Gamma_{1} & \ldots & \Gamma_{1} \\
\Gamma_{2} & I & \ldots & \Gamma_{2} \\
\vdots & \vdots & \ddots & \vdots \\
\Gamma_{n} & \Gamma_{n} & \ldots & I
\end{array}\right)
$$

is a bijection of the space $\mathcal{X}^{n}$, then $I-G$ is a bijection of the space $\mathcal{X}$.

Second. Let linear operators $\gamma_{i j}$ in $\mathcal{X}$ be defined as solutions of the equation

$$
\boldsymbol{\Phi} \cdot \boldsymbol{\Gamma}=\operatorname{diag}\left(\Gamma_{1}, \ldots \Gamma_{n}\right),
$$


where

$$
\boldsymbol{\Gamma}=\left(\begin{array}{cccc}
\gamma_{11} & \gamma_{12} & \ldots & \gamma_{1 n} \\
\gamma_{21} & \gamma_{22} & \ldots & \gamma_{2 n} \\
\vdots & \vdots & \ddots & \vdots \\
\gamma_{n 1} & \gamma_{n 2} & \ldots & \gamma_{n n}
\end{array}\right)
$$

and

$$
\operatorname{diag}\left(\Gamma_{1}, \ldots \Gamma_{n}\right)=\left(\begin{array}{cccc}
\Gamma_{1} & 0 & \ldots & 0 \\
0 & \Gamma_{2} & \ldots & 0 \\
\vdots & \vdots & \ddots & \vdots \\
0 & 0 & \ldots & \Gamma_{n}
\end{array}\right)
$$

Then the operator $\Gamma$ defined by

$$
I-\Gamma=(I-G)^{-1},
$$

is given in terms of the $\gamma_{i j}$ by the formula

$$
\Gamma=\sum_{\substack{1 \leq i \leq n \\ 1 \leq j \leq n}} \gamma_{i j}
$$

Note that the following identities are valid:

$$
\gamma_{i j}=\Gamma_{i}\left(\delta_{i j} I-\sum_{k \neq i} \gamma_{k j}\right)=\left(\delta_{i j} I-\sum_{k \neq j} \gamma_{i k}\right) \Gamma_{j} .
$$

Third. The components $\gamma_{i j}$ can be recovered by the operator $\Gamma$ by the formulas

$$
\gamma_{i j}=-\delta_{i j} G_{j}-G_{i}(I-\Gamma) G_{j} .
$$

Finally, we make a remark that will be important in what follows. Put $\boldsymbol{\Phi}=I+\boldsymbol{\Delta}$. Then

$$
\mathbf{\Phi}^{-1}=(I+\boldsymbol{\Delta})^{-1}=\left(I-\boldsymbol{\Delta}^{2}\right)^{-1}(I-\boldsymbol{\Delta}) .
$$

The components of the operator matrix $\Delta^{2}$ are sums of operators of the form $\Gamma_{i} \Gamma_{j}$ $(i \neq j)$. If the operator $\boldsymbol{\Delta}$ is bounded in an appropriate Banach space and the norm of $\Delta^{2}$ is less than 1 , then

$$
\Gamma=\sum \Gamma_{i}-\sum_{i \neq j} \Gamma_{i} \Gamma_{j}+\ldots
$$

where the series of sums converges in norm.

Step 4: separation of contributions. As has already been mentioned, the conjugation problem

$$
S_{+}=S_{-} W
$$

is equivalent to the inversion problem for the operator $I-F^{T} q$ with the multiplication operator $F=I-W$.

We employ the approach described above (the Schwarz method), putting $G_{j}=F_{j}^{T} q$. Observe that for $i \neq j$ the kernel of the product $G_{i} G_{j}$ is a diagonal matrix-valued function with diagonal entries of the form

$$
\int_{\mathbb{R}} d z \frac{g_{i}(x) e^{i t \varkappa_{i}\left(x-k_{i}\right)^{2}}}{(x-z)} \cdot \frac{g_{j}(z) e^{i t \varkappa_{j}\left(z-k_{j}\right)^{2}}}{(z-y-i 0)}=e^{i t \varkappa_{i}\left(x-k_{i}\right)^{2}} \chi_{i}(x) O\left(t^{-1 / 2}(1+|y|)^{-1}\right),
$$

where the functions $g_{i}$ and $g_{j}$ are smooth and have disjoint supports (controlled by the cut-off functions $\chi_{i}$ and $\chi_{j}$ ) lying in neighborhoods of the points $k_{i}$ and $k_{j}$, respectively. 
Since the contributions of the stationary points are localized, the role of the space $\mathcal{X}$ in the Schwarz method can be played by the space $H^{\alpha}=H^{\alpha, 0}(\mathbb{R})$ of Hölder functions on the axis with an exponent $\alpha$ close to zero. It is well known that the singular operators $p$ and $q$ are bounded in $H^{\alpha}$. As a consequence, the operators $G_{i}$ and $\Gamma_{i}$ will be bounded with norm of order of $O\left(t^{\alpha}\right)$. The operator products $G_{i} G_{j}, G_{i} \Gamma_{j}, \Gamma_{i} G_{j}, \Gamma_{i} \Gamma_{j}$ will have small norm of order of $O\left(t^{\alpha-1 / 2}\right)$, which is implied by the above estimate for the kernel of the product $G_{i} G_{j}$.

Consequently, in this leading order in $t$ we have

$$
\Gamma=\sum \Gamma_{j}+\sum_{i \neq j} \Gamma_{i} \Gamma_{j}+O\left(t^{\alpha-1}\right)
$$

and

$$
\begin{aligned}
K^{T} & =(I-\Gamma) \sum F_{i}^{T}=\left(I-\sum \Gamma_{j}\right) \sum F_{i}^{T}+\sum_{j \neq i} \Gamma_{j} \Gamma_{i} \sum F_{k}^{T}+O\left(t^{\alpha-1}\right) \\
& =\sum\left(I-\Gamma_{i}\right) F_{i}^{T}-\sum_{i \neq j} \Gamma_{j} F_{i}^{T}+\sum_{j \neq i ; k} \Gamma_{j} \Gamma_{i} F_{k}^{T}+O\left(t^{\alpha-1}\right) \\
& =\sum K_{j}^{T}+\mathcal{E}+O\left(t^{\alpha-1}\right),
\end{aligned}
$$

where we have put

$$
\mathcal{E}=-\sum_{i \neq j} \Gamma_{j} F_{i}^{T}+\sum_{j \neq i ; k} \Gamma_{j} \Gamma_{i} F_{k}^{T} .
$$

We need to estimate $p(\mathcal{E})$ in the norm of $H^{\alpha}$, see (2.18). For definiteness, we estimate the contribution of the term $\mathcal{E}_{i j}=\Gamma_{j} F_{i}^{T}$. Observe that

$$
-\Gamma_{j} F_{i}^{T}=\left(I-\Gamma_{j}\right) G_{j} F_{i}^{T}=K_{j}^{T} q F_{i}^{T} .
$$

This function is of the form

$$
e^{i t \varkappa_{j}\left(x-k_{j}\right)^{2}} \chi_{j}(x) O\left(t^{-1 / 2}\right),
$$

where, viewed as a function of $x, O\left(t^{-1 / 2}\right)$ is differentiable without distorting the order in $t$. Therefore, as soon as the argument of the function $p\left(\mathcal{E}_{i j}\right)$ goes beyond the domain controled by the cut-off function $\chi=\sum \chi_{j}$, we can use the stationary phase method to obtain an estimate of the form $O\left(t^{-1}\right)$ for the contribution in question. The contributions of the other terms are similar.

Thus, up to quantities of order of $O\left(t^{\alpha-1}\right)$, where $\alpha$ can be takes as close to zero as we wish, the contribution to $K^{T}$ of the stationary points of the phase function is additive.

Step 5: moving backward. To obtain explicit formulas, for us it will be convenient to return from factorization of the matrices

$$
\left(\begin{array}{cc}
1 & f_{j} T_{j}^{-1} \\
h_{j} T_{j} & 1
\end{array}\right)
$$

to that of the matrices of the form

$$
\left(\begin{array}{cc}
1+m_{j} n_{j} P & m_{j} T_{j}^{-1} \\
n_{j} T_{j} & 1+m_{j} n_{j} Q
\end{array}\right) .
$$

The reason is that, as was mentioned above, in the leading order, the asymptotics of the factorizing factors only depends on the asymptotics of the functions $m_{j}, n_{j}$ at the point $k_{j}$. Factorizing the diagonal of the last-written matrix, we get the following equation for $m_{j}, n_{j}$ :

$$
\left(\begin{array}{cc}
s_{j-} & 0 \\
0 & t_{j-}
\end{array}\right)\left(\begin{array}{cc}
1 & f_{j} T_{j}^{-1} \\
h_{j} T_{j} & 1
\end{array}\right)\left(\begin{array}{cc}
s_{j+} & 0 \\
0 & t_{j+}
\end{array}\right)=\left(\begin{array}{cc}
1+m_{j} n_{j} P & m_{j} T_{j}^{-1} \\
n_{j} T_{j} & 1+m_{j} n_{j} Q
\end{array}\right)
$$


where $s_{j+} s_{j-}=1+m_{j} n_{j} P$ and $t_{j+} t_{j-}=1+m_{j} n_{j} Q$. We have

$$
f_{j} s_{j-} t_{j+}=m_{j}, \quad h_{j} s_{j+} t_{j-}=n_{j},
$$

whence

$$
f_{j} h_{j}\left(1+m_{j} n_{j}\right)=m_{j} n_{j}
$$

i.e.,

$$
m_{j} n_{j}=\frac{f_{j} h_{j}}{1-f_{j} h_{j}}
$$

which agrees with the previous relations and determines the $m_{j}, n_{j}$.

Similarly, we get

Consequently,

$$
f_{j} h_{j}=\frac{\gamma_{j} \delta_{j} \chi_{j}^{2}}{1+\sum \gamma_{k} \delta_{k} \chi_{k}^{2}}
$$

$$
m_{j} n_{j}=\frac{\gamma_{j} \delta_{j} \chi_{j}^{2}}{1+\sum_{i \neq j} \gamma_{i} \delta_{i} \chi_{i}^{2}}=\gamma_{j} \delta_{j} \chi_{j}^{2}
$$

whence $\varphi_{j \pm}=s_{j \pm}$ and $\psi_{j \pm}=t_{j \pm}$. But this means that $m_{j}=\gamma_{j} \chi_{j}$ and $n_{j}=\delta_{j} \chi_{j}$. Thus, we arrive at factorization problems for the following matrix products:

$$
M_{j-}^{-1} M_{j+}=\left(\begin{array}{cc}
\varphi_{j-}^{-1} & 0 \\
0 & \psi_{j-}^{-1}
\end{array}\right)\left(\begin{array}{cc}
1+\gamma_{j} \delta_{j} \chi_{j}^{2} P & \gamma_{j} \chi_{j} T_{j}^{-1} \\
\delta_{j} \chi_{j} T_{j} & 1+\gamma_{j} \delta_{j} \chi_{j}^{2} Q
\end{array}\right)\left(\begin{array}{cc}
\varphi_{j+}^{-1} & 0 \\
0 & \psi_{j+}^{-1}
\end{array}\right) .
$$

Putting $N_{j \pm}=M_{j \pm}-I$, we see that

$$
H_{+} \sim\left[I+\sum N_{j+}\right] \Psi_{+} R_{+} \Theta_{+}^{-1}, \quad H_{-} \sim\left[I+\sum N_{j-}\right] \Psi_{-}^{-1} L_{-}^{-1} \Theta_{-} .
$$

As a result, we have the following statement.

Theorem 3.1. For sufficiently large $t>0$, the conjugation problem in question has a solution whose asymptotics has the form (3.21) with an errow of $O\left(t^{\varepsilon-1}\right)$, with $\varepsilon>0$ arbitrarily small, relative to $H^{\alpha, 1}(\mathbb{R})$, where $\alpha>0$ is sufficiently small.

3.3. Asymptotics of the off-diagonal entries of the matrix $H$ at large $|x|$. As we have seen, the asymptotic behavior of the matrix $H$ in the scattering domain is determined by that of its off-diagonal entries. At the same time, the asymptotics of the factors $\Psi_{ \pm}, L, R$, and $\Theta_{ \pm}$as $x \rightarrow \infty$ show that the asymptotics of the off-diagonal etries of $H$ is determined by the corresponding element of $\sum N_{j}$. With the same accuracy as above, we find

$$
N_{j \pm} \sim O_{j \pm}
$$

where the matrices $O_{j \pm}$ are given by the analytic factorization

$$
\left(I+O_{j+}\right)=\left(I+O_{j-}\right)\left(\begin{array}{cc}
1+\gamma_{j} \delta_{j} P_{j} & \gamma_{j} T_{j}^{-1} \\
\delta_{j} T_{j} & 1+\gamma_{j} \delta_{j} Q_{j}
\end{array}\right) .
$$

However, this factorization problem was solved in the paper [1] devoted to the case of one turning point (see \$2). We use formula (2.17), replacing, in accordance with (3.7), $a_{0}, d_{0}, \Delta_{0}$ by $a_{j}, d_{j}, \Delta_{j}, b_{0}$ and $c_{0}$ by $b_{j} e^{-i t \Phi\left(k_{j}\right)}$ and $c_{j} e^{i t \Phi\left(k_{j}\right)}$; and $t$ by $t\left|\Phi^{\prime \prime}\left(k_{j}\right)\right|$. Also, we introduce the following indicator of a turning point:

$$
\varkappa_{j}=\operatorname{sgn} \Phi^{\prime \prime}\left(k_{j}\right) \text {. }
$$

Then, by symmetry, the asymptotics of $H_{12}$ as $x, t \rightarrow \infty$ is given by

$$
H_{12} \sim \sum \frac{e^{-i t \Phi\left(k_{j}\right)} a_{j} d_{j}}{c_{j} \Delta_{j}} \frac{\sqrt{2 \pi}}{\Gamma\left(-i \nu_{j} \varkappa_{j}\right)}\left(t\left|\Phi^{\prime \prime}\left(k_{j}\right)\right|\right)^{-\frac{1}{2}-i \nu_{j} \varkappa_{j}} e^{-\left(A_{\varkappa_{j} j}+B_{-\varkappa_{j} j}\right) \varkappa_{j}} e^{-i \frac{\pi}{4} \varkappa_{j}} e^{-\frac{\pi \nu_{j}}{2}} \cdot \frac{\varkappa_{j}}{x} .
$$


The change of the sign (the coefficient $\varkappa_{j}$ of $1 / x$ ) for the contribution of a maximum point is explained naturally by the change of the direction of decreasing compared to the case of a point of minimum. We can also observe that, passing to the complex conjugate conjugation matrix, we get a conjugation problem of the form

$$
\widetilde{H}_{-}=\widetilde{H}_{+} \bar{V} .
$$

The inverse to the matrix $\bar{V}$ is equal to

$$
\frac{1}{\bar{\Delta}}\left(\begin{array}{cc}
\bar{d} & -\bar{b} T \\
-\bar{c} T^{-1} & \bar{a}
\end{array}\right) .
$$

In essence, this means that a problem with a maximum point reduces to that with a minimum point if we replace $b$ and $c$ with $-b$ and $-c$, which, once again, leads to formula (3.23).

\section{§4. Applications}

4.1. Modified Korteweg-de Vries equation. As an example, here we briefly discuss calculation of the asymptotics for the solution of the Cauchy problem for a well-known nonlinear equation of mathematical physics, namely, the modified Korteweg-de Vries equation.

This equation has the form

$$
u_{t}-6 u^{2} u_{x}+u_{x x x}=0
$$

with the following initial condition:

$$
\left.u\right|_{t=0} \text { is a smooth function decaying rapidly as } x \rightarrow \infty .
$$

The matrix conjugation problem associated with this equation (see, e.g., 4]) has the standard form described above:

$$
H_{+}=H_{-} V, \quad V(k)=\left(\begin{array}{cc}
1-|r(k)|^{2} & -\overline{r(k)} e^{-i t \Phi(k)} \\
r(k) e^{i t \Phi(k)} & 1
\end{array}\right),
$$

with the phase function

$$
\Phi(k)=8 k^{3}+2 l k,
$$

where $l$ has the meaning of the ratio $\frac{x}{t}$. The reflection coefficient $r(k)$ satisfies

$$
\overline{r(-k)}=-r(k) \text {. }
$$

The relationship between the solution of the above Cauchy problem and the solution of the corresponding auxiliary matrix Riemann problem is given by the formula

$$
u(x, t)=2 \lim _{k \rightarrow \infty} k H_{12}(k) .
$$

The derivative $\Phi^{\prime}$ has the roots $k_{1.2}= \pm k_{0}, k_{0}=\sqrt{-\frac{l}{12}}$ (the square root is arithmetical). We have

$$
\Phi\left(k_{1}\right)=-\Phi\left(k_{2}\right)=-16 k_{0}^{3}, \quad \Phi^{\prime \prime}\left(k_{1}\right)=-\Phi^{\prime \prime}\left(k_{2}\right)=48 k_{0} .
$$

Next, we put

$$
r\left(k_{1}\right)=r_{0}, r\left(k_{2}\right)=r\left(-k_{1}\right)=-\overline{r\left(k_{1}\right)}=-\overline{r_{0}}, \quad \nu_{1}=\nu_{2}=\nu_{0} \equiv \frac{-1}{2 \pi} \ln \left(1-\left|r_{0}\right|^{2}\right) .
$$


Finally, to compute $B_{1}$ and $B_{2}$ we observe that

$$
\begin{aligned}
& \frac{-1}{2 \pi i} \int_{-k_{0}}^{k_{0}} \frac{-\ln \left(1-|r(y)|^{2}\right) d y}{x-y+i 0} \\
& \quad=\left.\frac{i}{2 \pi} \ln \left(1-|r(y)|^{2}\right) \ln (x-y+i 0)\right|_{y=-k_{0}} ^{y=k_{0}}-\frac{i}{2 \pi} \int_{-k_{0}}^{k_{0}} \ln (x-y+i 0) d \ln \left(1-|r(y)|^{2}\right) .
\end{aligned}
$$

Assuming that $\ln (x+i 0)$ is defined as the principal branch on the plane with cut along the positive semiaxis and letting $x \rightarrow k_{0}$, we obtain

$$
\begin{aligned}
\frac{-1}{2 \pi i} \int_{-k_{0}}^{k_{0}} & \frac{-\ln \left(1-|r(y)|^{2}\right) d y}{x-y+i 0} \\
& \sim-i \nu_{0} \ln \left(x-k_{0}+i 0\right)+i \nu_{0} \ln \left(2 k_{0}\right)-\frac{i}{2 \pi} \int_{-k_{0}}^{k_{0}} \ln \left|k_{0}-y\right| d \ln \left(1-|r(y)|^{2}\right) .
\end{aligned}
$$

This means that

$$
\begin{aligned}
A_{1+} & =-i B+i \nu_{0} \ln \left(2 k_{0}\right), \\
B & =\frac{1}{2 \pi} \int_{-k_{0}}^{k_{0}} \ln |k-y| d \ln \left(1-|r(y)|^{2}\right), \quad B_{1-}=A_{1+}-2 \pi \nu_{0} .
\end{aligned}
$$

Letting $x \rightarrow-k_{0}$, we see that

$$
\begin{aligned}
& \frac{-1}{2 \pi i} \int_{-k_{0}}^{k_{0}} \frac{-\ln \left(1-|r(y)|^{2}\right) d y}{x-y+i 0} \\
& \quad \sim i \nu_{0} \ln \left(x+k_{0}+i 0\right)-i \nu_{0} \ln \left(-2 k_{0}+i 0\right)-\frac{i}{2 \pi} \int_{-k_{0}}^{k_{0}} \ln \left(-k_{0}-y+i 0\right) d \ln \left(1-|r(y)|^{2}\right) \\
& \quad=i \nu_{0} \ln \left(x+k_{0}+i 0\right)-i \nu_{0} \ln \left(2 k_{0}\right)+\pi \nu_{0}-\frac{i}{2 \pi} \int_{-k_{0}}^{k_{0}} \ln \left|k_{0}+y\right| d \ln \left(1-|r(y)|^{2}\right) \\
& \quad=i \nu_{0} \ln \left(x+k_{0}+i 0\right)-i \nu_{0} \ln \left(2 k_{0}\right)+\pi \nu_{0}+\frac{i}{2 \pi} \int_{-k_{0}}^{k_{0}} \ln \left|k_{0}-y\right| d \ln \left(1-|r(y)|^{2}\right)
\end{aligned}
$$

whence

$$
A_{2+}=-A_{1+}+\pi \nu_{0}
$$

which implies

$$
A_{2-}=A_{1+}+\pi \nu_{0}, \quad B_{2+}=A_{1-}+\pi \nu_{0} .
$$

Thus, for the asymptotics of the solution of the Cauchy problem in the case of the modified Korteweg-de Vries equation we find

$$
\begin{aligned}
& u \sim 2\left[\frac{e^{i 16 t k_{0}^{3}}}{r_{0}} \frac{\sqrt{2 \pi}}{\Gamma\left(-i \nu_{0}\right)}\left(48 k_{0} t\right)^{-\frac{1}{2}-i \nu_{0}} e^{2 i B-2 i \nu_{0} \ln \left(2 k_{0}\right)} e^{-i \frac{\pi}{4}} e^{-\frac{\pi \nu_{0}}{2}}\right. \\
&\left.-\frac{e^{-i 16 t k_{0}^{3}}}{-\overline{r_{0}}} \frac{\sqrt{2 \pi}}{\Gamma\left(i \nu_{0}\right)}\left(48 k_{0} t\right)^{-\frac{1}{2}+i \nu_{0}} e^{-2 i B+2 i \nu_{0} \ln \left(2 k_{0}\right)} e^{i \frac{\pi}{4}} e^{-\frac{\pi \nu_{0}}{2}}\right]
\end{aligned}
$$

$=A \cos \Psi$,

$$
\begin{aligned}
A & =\frac{4 \sqrt{2 \pi}}{\left|r_{0}\right|\left|\Gamma\left(-i \nu_{0}\right)\right| \sqrt{48 k_{0} t}} e^{-\frac{\pi \nu_{0}}{2}}=\sqrt{\frac{\nu_{0}}{3 k_{0} t}}, \\
\Psi & =16 t k_{0}^{3}-\nu_{0} \ln \left(48 k_{0} t\right)-\arg \Gamma\left(-i \nu_{0}\right)-\arg r_{0}+2 B-2 \nu_{0} \ln \left(2 k_{0}\right)-\frac{\pi}{4} \\
& =16 k_{0}^{3} t-\nu_{0} \ln t-\nu_{0} \ln \left(192 k_{0}^{3}\right)+\arg \Gamma\left(i \nu_{0}\right)-\arg r_{0}+2 B-\frac{\pi}{4}
\end{aligned}
$$


4.2. Remarks on comparison of approaches. For the first time, the strict justification of the asymptotics for the solution of the Cauchy problem for the modified Korteweg-de Vries equation, based on its relationship with the conjugation problem, was obtained in [5. The authors of [5, 6] called their approach to exploring the asymptotics of soltutions of the corresponding conjugation problems with oscillating matrices the fastest descent method. The main part of their calculations consists in solution of a series of auxiliary Riemann problems on cross-like contours. This idea is indeed borrowed from the standard steepest descent method. However, the relationship between the Riemann problems mentioned above is rather tricky. The difficulties related to this approach seem to be quite predictable. For the steepest descent method to be applicable to calculation of the asymptotics of the integral

$$
\int_{\Gamma} F(z) e^{i t \Phi(z)} d z
$$

it is necessary that the functions $F$ and $\Phi$ be analytic. Change of variables can help in the case of a nonanalytic $\Phi$, but the nonanalyticity of $F$ is more important.

The choice in favor of the stationary phase method, made by V. C. Buslaev and the author of the present paper, makes it possible to avoid the above-mentioned difficulties under the approach of Deift and Zhou.

\section{REFERENCES}

[1] A. M. Budylin and V. S. Buslaev, Quasiclassical asymptotics of the solutions of matrix RiemannHilbert problems with quadratic oscillation of non-diagonal elements, Funktsional. Anal. i Priložen., 2013 (Russian) (to appear).

[2] A. M. Budylin and V. S. Buslaev, Quasiclassical asymptotic of the resolvent of an integral convolution operator with a sine kernel on a finite interval, Algebra i Analiz 7 (1995), no. 6, 79-103; English transl., St. Petersburg. Math. J. 7 (1996), no. 6, 925-942. MR1381979 (97b:47050)

[3] I. Ts. Gokhberg and N. Ya. Krupnik, Introduction to the theory of one-dimensional singular integral operators, Izdat. Stiinca, Kishinev, 1973. (Russian) MR0405177 (53:8971)

[4] P. A. Deift, A. R. Its, and X. Zhou, Long-time asymptotics for integrable nonlinear wave equations, Important developments in soliton theory, 181-204, Springer Sci. Nonlinear Dynam., Springer, Berlin, 1993. MR 1280475 (95h:35031)

[5] P. A. Deift and X. Zhou, A steepest descent method for oscillatory Riemann-Hilbert problems. Asymptotics for the MKdV equation, Ann. of Math. (2) 137 (1993), no. 2, 295-368. MR1207209 (94d:35143)

[6] , Long-time behavior of the non-focusing nonlinear Schrödinger equation - a case study, Univ. of Tokyo, Tokyo, 1994.

[7] G. G. Varzugin, Asymptotics of oscillatory Riemann-Hilbert problems, J. Math. Phys. 37 (1996), no. 11, 5869-5892. MR1417182 (97i:30055)

[8] Do Yen, A nonlinear stationary phase method for oscillatory Riemann-Hilbert problems, arXiv: 0910.2533v2 [math.CA], 2010. MR2806592(2012e:37137)

Department of Physics, St. Petersburg State University, Ul'yanovskaya 3, Petrodvorets, St. Petersburg 185504, Russia

E-mail address: budylin@math.nw.ru

Received 26/OCT/2012

Translated by A. PLOTKIN 